\title{
Investigation of Investment Casting Pattern Using Fused Deposition Modeling
}

\author{
Kamal Ukey $^{\circledR}$, Santosh Hiremath ${ }^{\circledR}$, Himadri Majumder ${ }^{*}$ \\ G H Raisoni College of Engineering and Management, Pune, India \\ E-mail: himu.nita@gmail.com
}

Received: 6 May 2021; Revised: 25 June 2021; Accepted: 20 July 2021

\begin{abstract}
In today's economic climate, various organizations fight with decreasing sales and increasing costs. However, industries that have implemented the process of investment casting are one of the ways of manufacturing complex metallic parts at a low cost. High tooling costs and long manufacturing time are associated with the metal molds production for producing investment casting wax (sacrificial) patterns. It leads to a problem with cost justification for personalized single casting or production of small lots. The present study evaluates the suitability of the fused deposition modeling (FDM) fabricated pattern for investment casting. For this, a case study on a part was also conducted to collect experimental data regarding the process. A trial component was fabricated in an FDM machine and then cast by the investment method. This research resulted in reduced process time and cost for the small and medium size of the batch.
\end{abstract}

Keywords: fused deposition modeling (FDM), investment casting, rapid prototyping (RP), polylactic acid

\section{Introduction}

One of the oldest methods of metal forming is investment casting (IC) or lost wax casting. Various types of materials, with stainless steel alloys, brass, aluminum, and carbon steel, may be used for the investment casting process [1]. The conventional investment casting process chain is shown in Figure 1.

As can be seen, the process of making the wax pattern is very tedious and requires machinery and tooling to be accomplished.

On the other hand, fused deposition modeling (FDM) is a rapid prototyping process and uses polylactic acid (PLA) as a building material. For conventional wax pattern tooling, it can be considered as an alternative. According to this concept, the possibility of using PLA patterns is discussed and a case study is also present.

\section{Literature review}

The melt extrusion technique is used generally in FDM, where according to 3D model data, the material is deposited layer by layer until a whole object is completed. In FDM, filament having a diameter of less than $3 \mathrm{~mm}$ is used as feedstock into a printer through a circulating drive gear system. For moving the filament through the system

Copyright (C2021 Himadri Majumder, et al.

DOI: https://doi.org/10.37256/est.222021904

This is an open-access article distributed under a CC BY license

(Creative Commons Attribution 4.0 International License)

https://creativecommons.org/licenses/by/4.0/ 
stepper motor is linked to one of the ends of the gear drives [2]. Numerous FDM applications use plastics including acrylonitrile butadiene styrene (ABS) and polycarbonate (PC). Mentioned plastic patterns have been confirmed to be efficient and within tolerance in investment casting applications. Durability, clean burnout, capability to be controlled without destruction, structural strength, and easiness of pattern development are the main benefits of ABS as compare to other RP plastic patterns [3]. As per the previous study, the glass transition temperature is the most delicate property of material followed by the coefficient of thermal expansion and Young's modulus. A plastic material synthesizer can make better disposable plastics for investment casting [4]. In the investment casting process, the accuracy of wax patterns utilized in the IC process affects the accuracy of complete cast part which causes costly low volume production involved in prototyping of customized human implants or other specific element productions [5]. For two output parameters (surface roughness and dimensional accuracy), all three input parameters (metal type, volume/surface-area ratio, and layer's combination) play a very significant role [6]. A standard part utilized for the evaluation of the FDM process is comprised of various features, shapes, and geometries [7]. Applied RP-FDM technique to determine whatever dimensional variations happened during individual production stages, starting from the 3D pattern printing [8]. Singh et al. suggested an approach that eliminates the shell cracking problem in the FDM pattern based on the investment casting process [9].

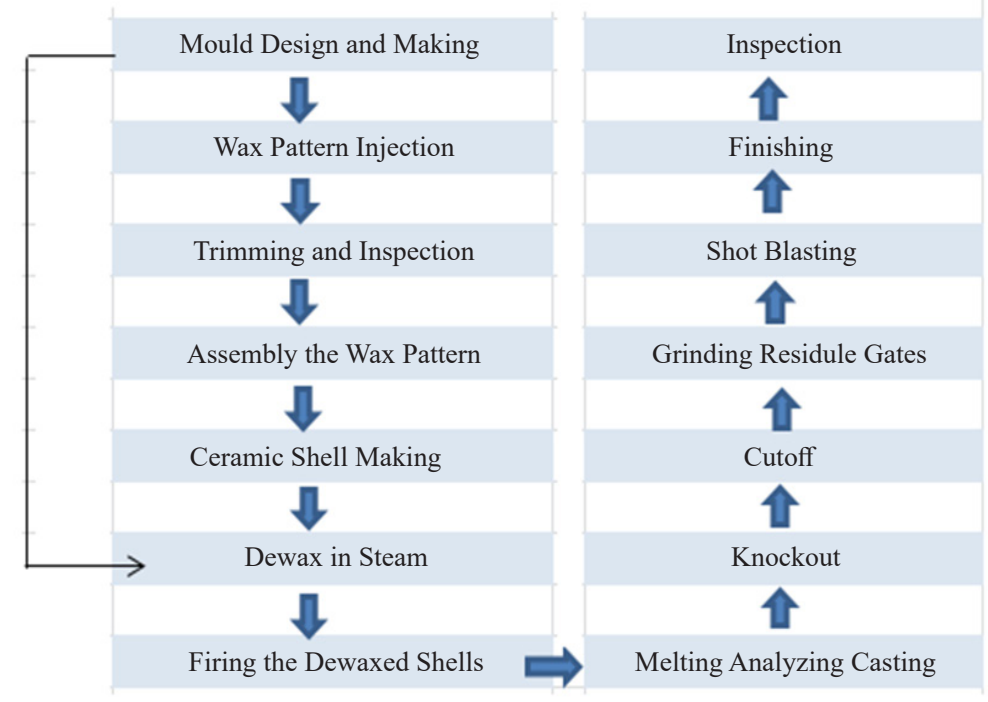

Figure 1. The process flow chart of Investment Casting

\section{Fused deposition modeling rapid prototyping}

Figure 2 presents in FDM process where the CAD model is saved in stereolithography (STL) file format. Scientifically slicing and arranging the model for build process support structures are automatically produced whenever required.

The FDM printer uses thermoplastics filaments, which are heated to the melting point, converted in a semiliquid form, and finally deposited by an extruder (layer by layer), and then utilizes a computer-aided design (CAD) file to create a final object. The layers of filament deposited by the extruder nozzle are fine as $0.04 \mathrm{~mm}$ thick, and the piece is built one layer at a time from the bottom up. FDM process is based on an additive principle where the material is deposited in layers. A metal wire or plastic filament is unwound from a coil and supplies material to an extrusion nozzle that can turn the flow on and off. To melt the material, the nozzle is heated and can be rotated in both horizontal and vertical directions by a numerically operated mechanism directly controlled by computer-aided manufacturing (CAM) software package. As the material hardens immediately after extrusion from the nozzle, the model or component is formed by extruding small beads of thermoplastic material to form different layers. Extruder 
uses servo or stepper driver [10].

There are many materials available with distinct strength-temperature trade-offs like ABS, PC, and waxes. Compared to tooling and machining, which needs multiple axes machining, the FDM machine has to deal with only 2 axes at a time, which makes any intricate, thin-walled, and complex shape possible.

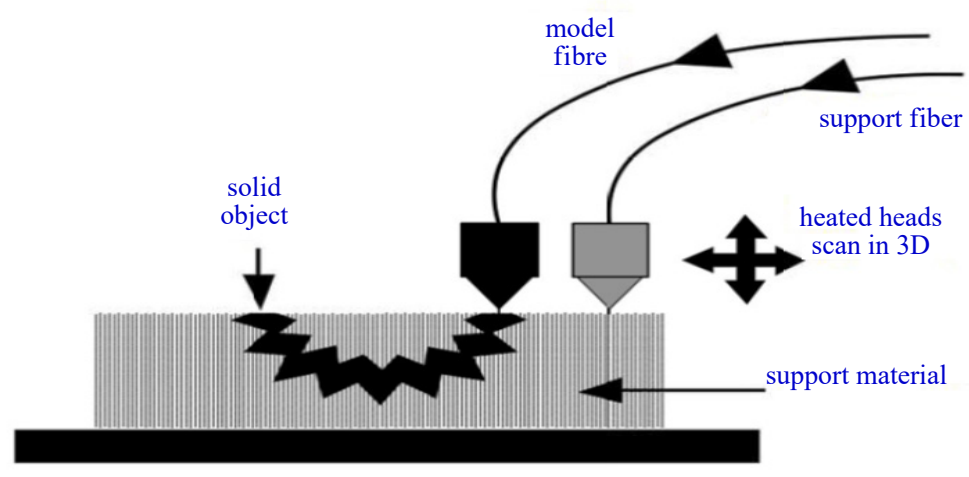

Figure 2. Schematic of the FDM process

\section{Procedure}

First of all, the pattern to be cast is 3D modeled in a CAD modeling package and then imported to 'Skeinforge' in STL format. As per the values given in Table 1 process parameters are set and then by using PLA parts are fabricated. Table 2 shows the material properties of the selected metal material for the IC pattern. If the shape were to be very complicated, two nozzles are used, one for the main part and the other to provide support for the material. Hence any complicated shape of the pattern is possible as the support material can be dissolved once the shape of the pattern sets. The 3D model of the component is shown in Figure 3.

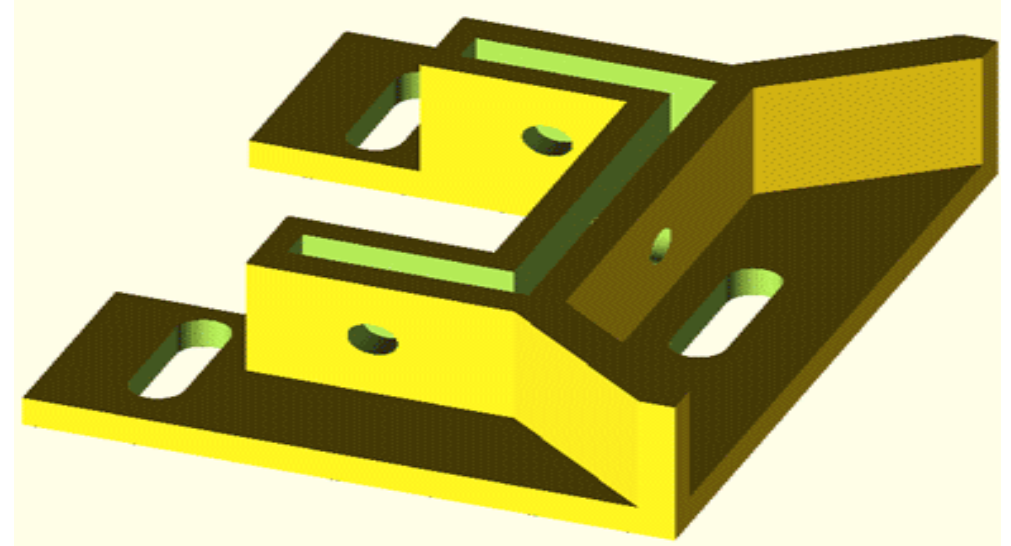

Figure 3. The 3D model 
Table 1. FDM process parameters

\begin{tabular}{ccc}
\hline Sr. no & Settings & Value \\
\hline 1 & Raster angle & 45 degree \\
2 & Air gap & $0.0 \mathrm{~mm}$ \\
3 & Slice thickness & $0.254 \mathrm{~mm}$ \\
4 & Feed rate & $30 \mathrm{~mm} / \mathrm{s}$ \\
\hline
\end{tabular}

Table 2. Aluminium 3003 properties

\begin{tabular}{ccc}
\hline Sr. no & Property & Value (MPa) \\
\hline 1 & Ultimate strength & 150 \\
2 & Yield strength & 145 \\
3 & Shear ultimate strength & 95 \\
4 & Fatigue endurance limit & 60 \\
\hline
\end{tabular}

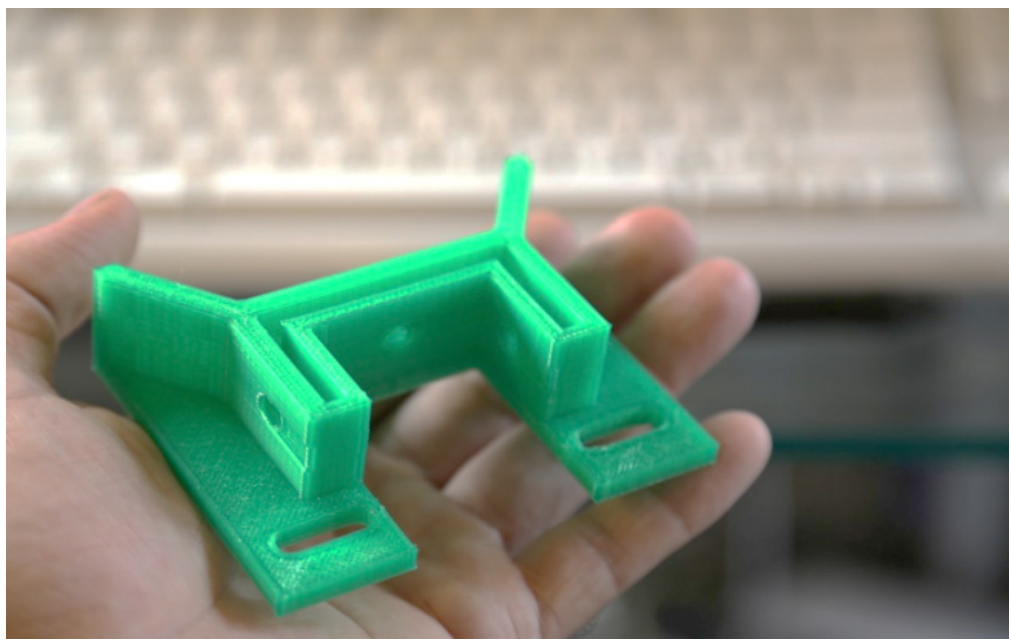

Figure 4. Rapid prototyped part in PLA

Figure 4 presents a rapid prototype in PLA in which part is designed considering ISO286-2 IT14 investment casting linear tolerance. Hence, the part is $103 \%$ of its original size considering aluminum [11]. Some finishing was done on the FDM fabricated part to improve its surface finish. Then the regular investment casting process was continued, gates and risers were attached to the pattern. The procedure slightly differs from a lost wax method, as PLA does not melt like wax. Hence, it is not kept for autoclaving. It has to be burnt out at a high temperature of above $650{ }^{\circ} \mathrm{C}$ after which only ash is left [12].

After the burnout process shown in Figure 5, the invested pattern has to be pressure cleaned with air to clear out the remaining ash left, which can affect the quality of the cast if not cleared out [13]-[15]. The molten aluminum which is shown in Figure 6, was then poured into the invested pattern. 


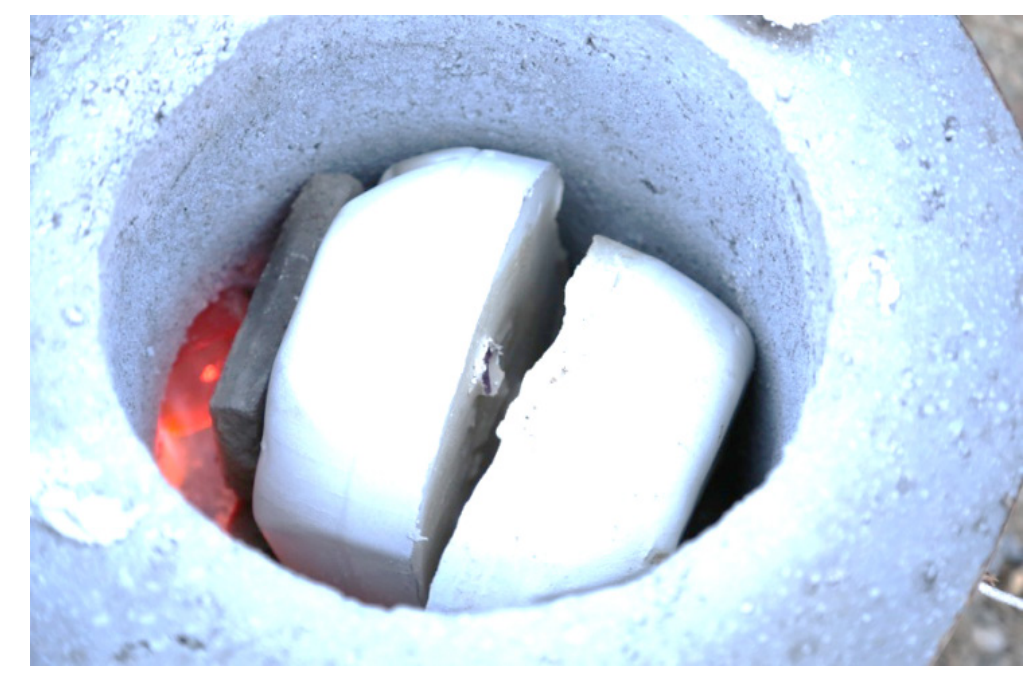

Figure 5. Burn out process

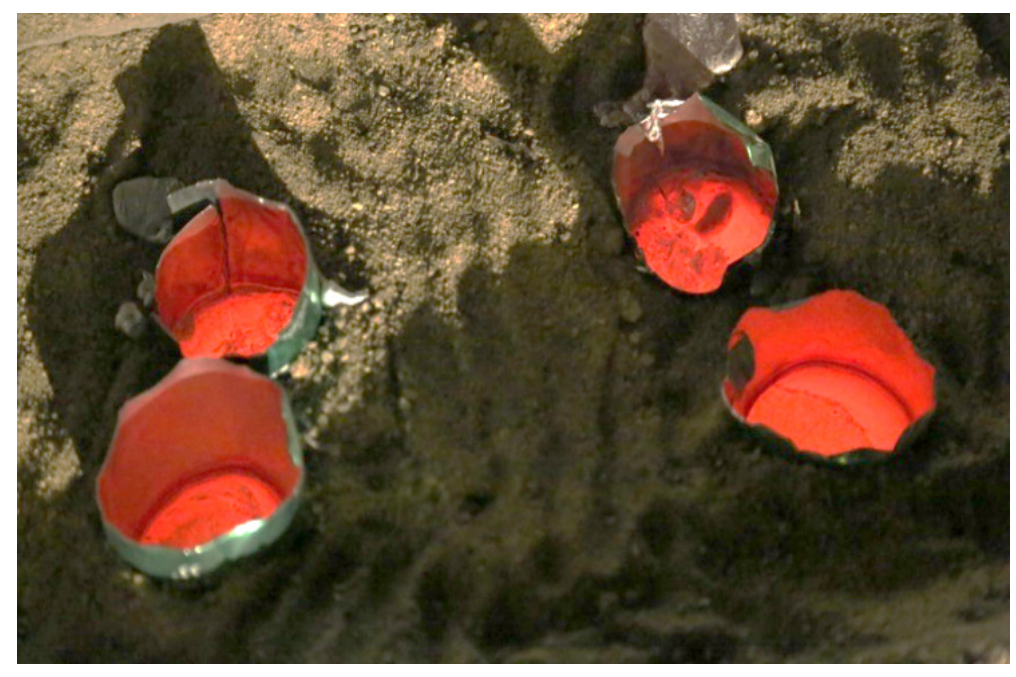

Figure 6. Molten metal poured

The pattern is allowed to cool off for a few hours after which the part is hammered out from the invested pattern by chipping off the investment. The gates and risers were cut off and the part was finished [16]-[18].

It found applications in the automobile industry like, develop and validate mufflers particularly the muffler for exhaust, which can give advantages over the traditional method with short development [19].

\section{Result}

The cast part was compared to the original part drawing and is shown in Table 3. After comparison, dimension parameters, like the thin wall width, thin-wall length, hole, slot, overall length, and overall width, are very close to the original value defined in part drawing and under the acceptance range.

The casting satisfies the dimensional tolerance set and no further machining is required. The finished part is shown in Figure 7. The surface finish was also found as 125 micro inch which is under satisfactory level. 
Table 3. Comparison of design dimensions with the cast part

\begin{tabular}{cccc}
\hline Sr. No & Parameter & $\begin{array}{c}\text { Design dimension } \\
(\mathrm{mm})\end{array}$ & $\begin{array}{c}\text { Measured dimension } \\
(\mathrm{mm})\end{array}$ \\
\hline 1 & Thin wall width & 3 & 2.98 \\
2 & Thin wall length & 27.07 & \\
3 & Hole & $\mathrm{R} 3$ & $12 * \mathrm{R} 2.5$ \\
4 & Slot & $12 * \mathrm{R} 2.5$ & 80.06 \\
5 & Overall length & 80 & 60.08 \\
6 & Overall width & 60 & \\
\hline
\end{tabular}

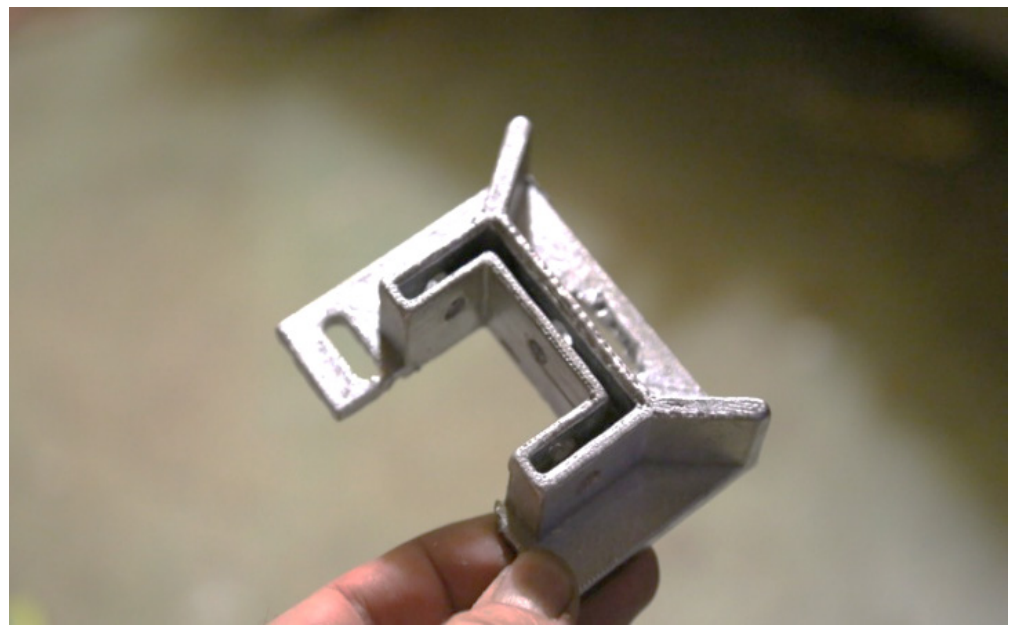

Figure 7. Finished part

\section{Conclusion}

With the rapid advances in manufacturing technology, FDM rapid prototyping process is considered and applied for investment casting and the following outcomes may be drowning:

1. The applicability of the FDM process in investment casting was investigated. A trial component was fabricated in an FDM machine and then cast by the investment casting method.

2. This technique will decrease the processing time by $40 \%$, also save $50 \%$ production cost for a small or medium size batch.

3. It is massive progress for components whose design keeps changing according to the situation for example turbocharger casing, unique fittings, special studs, bolts, etc.

4. In FDM we get defect-free products.

Further scope of development would be if the part has to be produced in a larger batch then the mold can be FDM fabricated and wax can be injection molded into it. This will also save a lot of time as the FDM fabricated PLA part need not be sacrificed for every cast. 


\section{Conflict of interest}

The authors declare no conflict of interest.

\section{References}

[1] S. Hiremath, K. Ukey, N. Kumar, and N. Gadlegaonkar, "The study of effect of speed process parameters in fused deposition modeling 3D printing for ABS material," International Journal of Engineering Research and Applications (IJERA), vol. 10, no. 6, pp. 20-24, 2020.

[2] T. N. A. T. Rahim, A. M. Abdullah, and H. M. Akil, "Recent developments in fused deposition modeling-based 3D printing of polymers and their composites," Polymer Reviews, vol. 59, no. 4, pp. 589-624, 2019.

[3] C. Gouldsen, and P. Blake, Investment Casting Using FDM/ABS Rapid Prototype Patterns. USA: Rapid ToolworX and Stratasys Inc., 1998, pp. 19.

[4] S. Wang, A. G. Miranda, and C. Shih, "A study of investment casting with plastic patterns," Materials and Manufacturing Processes, vol. 25, no. 12, pp. 1482-1488, 2010.

[5] R. Singh, and S. Singh, "Fused deposition modelling based rapid patterns for investment casting applications: A review," Rapid Prototyping Journal, vol. 22, no. 1, pp. 123-143, 2016.

[6] R. Singh, and S. Singh, "Effect of process parameters on surface hardness, dimensional accuracy and surface roughness of investment cast components," Journal of Mechanical Science and Technology, vol. 27, no. 1, pp. 191$197,2013$.

[7] A. Al-Ahmari, S. H. Mian, and W. Ameen, "Performance assessment of fused deposition modeling process," Journal of Engineering Research, vol. 9, no. 1, pp. 200-213, 2021.

[8] M. Mackü, and M. Horáček, "Applying RP-FDM technology to produce prototype castings using the investment casting method," Archives of Foundry Engineering, vol. 12, pp. 75-82, 2012.

[9] S. Singh, P. Kumar, and J. Singh, "An approach to eliminate shell cracking problem in fused deposition modeling pattern based investment casting process," InIOP Conference Series: Materials Science and Engineering, vol. 1091, no. 1, pp. 012035, 2021.

[10] O. A. Mohamed, S. H. Masood, and J. L. Bhowmik, "Optimization of fused deposition modeling process parameters: A review of current research and future prospects," Advances in Manufacturing, vol. 3, no. 1, pp. 42$53,2015$.

[11] K. A. Silva, InForce Rapid Proto Casting, Inc., Vancouver, WA USA 98685.

[12] D. Garlotta, "A literature review of poly (Lactic Acid)," Journal of Polymers and the Environment, vol. 9, pp. 6384, 2001.

[13] C. Gouldsen, and P. Blake, Investment Casting Using FDM/ABS Rapid Prototype Patterns. USA: Rapid Toolwor X and Stratasys Inc., 1998.

[14] P. M. Yuvraj, and K. Ukey, "Design and analysis of automobile exhaust system for-BS VI," International Research Journal of Engineering and Technology, vol. 7, no. 6, 2020.

[15] R. Singh, and G. Singh, "Investigations for statistically controlled investment casting solution of FDM-based ABS replicas," Rapid Prototyping Journal, vol. 20, no. 3, pp. 215-220, 2014.

[16] W. S. Harun, S. Safian, and M. H. Idris, "Evaluation of ABS patterns produced from FDM for investment casting process," Computational Method and Experiments in Materials Characterisation IV, vol. 10, no. 1, pp. 319-328, 2009.

[17] D. Singh, R. Singh, and K. S. Boparai, "Investigations on hardness of investment-casted implants fabricated after vapour smoothing of FDM replicas," Journal of the Brazilian Society of Mechanical Sciences and Engineering, vol. 42, no. 4, pp. 1-2, 2020.

[18] K. Tamta, and D. B. Karunakar, "Development of hybrid pattern material for investment casting process: An experimental investigation on improvement in pattern characteristics," Materials and Manufacturing Processes, vol. 6, pp. 1-8, 2020.

[19] Y. Xue, X. Wang, W. Wang, X. Zhong, and F. Han, "Compressive property of Al-based auxetic lattice structures fabricated by 3-D printing combined with investment casting," Materials Science and Engineering: A, vol. 11, no. 722, pp. 255-262, 2018. 